\title{
Estimation of the SARS-CoV-2 specific reproduction number in SAARC countries: A 60-days Data-driven analysis
}

\author{
Mohammed Nizam Uddin \\ Department of Applied Mathematics, Noakhali Science and Technology University, \\ Bangladesh \\ A.N.M. Rezaul Karim* \\ Department of Computer Science \& Engineering, International Islamic University Chittagong, \\ Bangladesh \\ Sofi Mahmud Parvez \\ Department of Applied Mathematics, Noakhali Science and Technology University, \\ Bangladesh \\ RiazMahmud \\ Department of Mathematics, South Asian University, India \\ H. M. Shahadat Ali \\ Department of Applied Mathematics, Noakhali Science and Technology University, \\ Bangladesh

\section{R. I. Faruque} \\ Space Science Centre (ANGKASA), Universiti Kebangsaan Malaysia, 43600 UKM, Bangi, \\ Selangor, Malaysia \\ *Corresponding Author. Email: zakianaser@yahoo.com
}

\section{How to Cite}

Uddin M. N. et al. (2020). Estimation of the SARS-CoV-2 specific reproduction number in SAARC countries: A 60-days Data-driven analysis. Journal of Applied and Natural Science, 12(4): 628 - 634. https://doi.org/10.31018/jans.v12i4.2397

\begin{abstract}
Novel coronavirus disease-2019 (COVID-19) was acknowledged as a global pandemic by WHO, which was first observed at the end of December 2019 in Wuhan city, China, caused by extreme acute respiratory syndrome coronavirus2 (SARS-CoV-2). According to the Weekly operation Update on COVID-19 (November 13, 2020) of the World Health Organization, more than 53 million confirmed cases are reported, including 1.3 million deaths. Various precautionary measures have been taken worldwide to reduce its transmission, and extensive researches are going on. The purpose of this analysis was to determine the initial number of reproductions (Ro) of the coronavirus of SAARC countries named Afghanistan, Bangladesh, India, Pakistan, Bhutan, Nepal, the Maldives, and Sri-Lanka for the first 60 days as the growth is exponential in the early 60 days. The reproduction numbers of coronavirus for Afghanistan, Bangladesh, India, Pakistan, Bhutan, the Maldives, Nepal, and Sri Lanka are 1.47, $3.86,2.07,1.43,1.31,3.22,1.75$, and 2.39 respectively. The basic reproduction number $\left(R_{0}\right) 3.86$ for Bangladesh and 1.31 for Bhutan indicated that up to 60-days of the outbreak COVID-19, the epidemic was more severe in Bangladesh and less severe in Bhutan among all the SAARC countries. Our predictions can be helpful in planning alertness and taking the appropriate measures to monitor it.
\end{abstract}

Keywords: Basic Number for reproduction, COVID-19, SAARC countries, SARS-CoV-2

\section{INTRODUCTION}

COVID-19 (coronavirus disease -2019) was first identified on December 31, 2019, from a person in Hubei province, China which is now declared a global pandemic by World Health Organization (WHO) (Liu et al., 2020). From the Weekly operation update on COVID19 (November 13, 2020) of the WHO, approximately $53,164,803$ confirmed cases and 1,300,576 deaths were identified globally. Still, now the coronavirus infection and mortality rates have been exponentially rising globally. No vaccine has yet been fully developed to protect against the virus. According to the update on November 15, 2020, of world meter (COVID-19 CORONAVIRUS PANDEMIC), the United States of America was the most affected country with $11,235,666$ confirmed cases and 6,891,461 deaths. India is the second most affected and deadliest country, including 8,834,331 cases and 129,914 deaths globally after the United States of America (https:// www.worldometers.info/ COVID-19 CORONAVIRUS PANDEMIC; last updated November 15, 2020). South 
Asian countries are one of the world's most heavily populated areas with a high population growth rate. The effects of coronavirus have been more worsened in South Asian countries than in other countries. (Hasan, 2020) Among the South Asian country region, Pakistan was the first country where confirmed coronavirus case was officially identified. (Dey et al., 2020). Bangladesh is the most affected country in the South Asian area, with over 432,333 confirmed cases and 6,194 , deaths, followed by Pakistan (407, 838 cases and 7,141 deaths), Nepal (209,776 cases and 1221 deaths), Afghanistan (43,240 cases and 1617 deaths), Sri-Lanka (17,287 cases and 58 deaths), and the Maldives (12,154 cases and 43 deaths) based on data up to November 15, 2020 (<https://www.worldomet ers.info/> COVID-19 CORONAVIRUS PANDEMIC; last updated November 15, 2020). The initial concept of an infectious disease related to an epidemic outbreak is the number of early reproductions. To understand the conduction dynamics of COVID-19, many epidemiological studies have been conducted, which are qualified in the most crucial primary reproduction number $\left(R_{0}\right)$ indicate the susceptibility of the various to completely innocent people, the expected number of new infections developed by the infected person is described (Heesterbeek, 2002). Our study focuses on the primary reproduction number of COVID-19. It has shown the range from 1.00 to 4.00 and initial identification during the early stages of the pandemic in South Asian areas, especially SRRAC countries. The present study has reported the basic reproduction number of COVID-19 in SRRAC included countries that will predict the transmission dynamics of COVID-19.

\section{MATERIALS AND METHODS}

Source of data : We gathered the daily confirmed number of covid-19 cases of time series data in world meter (coronavirus pandemic) for the first 60 days (https://www.worldometers.info/ COVID-19 CORONAVIRUS PANDEMIC; last update November 15 2020). In this analysis, we used the early 60 days of daily new cases where the number of cases is exponential.

Approximation of the basic reproduction number Ro: The concept of primary reproductive number $\left(R_{0}\right)$ is a vital parameter in epidemiology. In epidemiology, $\left(R_{0}\right)$ is defined by the number of secondary cases that occurred by an infected person over his entire times of infectiousness in an absolutely susceptible population. If $R_{0}>1$, not more than one in each existing infection. In this situation, the virus can shrink and ultimately die. If $R_{0}=1$, A new condition occurs for each established infection. The virus may stay active and stable, but there will be no epidemic or plague. And if it $R_{0}>1$ is, it Causes multiple infections for each current infection (Mustafa, F et al. I,2020). There are various methods for calculating the estimation of Ro, such as Exponential Growth (EG), Sequential Bayesian (SB), Maximum Likelihood (ML), the Time-Dependent (TD), and so on. In our analysis, we used the EG method for calculating the basic reproduction number in $R_{0}>1$ SRRAC countries.

Exponential growth (EG): In the early stage of the disease outbreak, the reproductive rate was connected to the exponential Growth rate. As reported by Wallinga and Lipsitch (2007), the basic reproduction number is described as

$$
R=\frac{1}{M(-r)}
$$

where the exponential rate of growth is symbolized by $r$, which is represented by a per capita change in the number of new cases per unit of time, and $M$ stands for the moment generating function of the generation time distribution

Ro package: For calculation of the basic number of reproductions Ro and analyzing the data, have been used $R$ software (version 4.0.2) including the Ro package (Obadia et al., 2012).

R script: library(R0)

Country $=c$ (input data)

names(country)<-seq(from=as.Date("start"),

to=as.Date("End"), by=1)

mGT=generation.time("gamma",c(5.2,1.72))

plot(country)

sen=sensitivity.analysis(sa.type="time", incid=country, $G T=m G T$, begin=1:30, end=31:60, est.method="EG")

plot(sen, what=c("criterion","heatmap"))

est.RO.EG(Country, $\mathrm{mGT}$, begin = ,end $=$ )

\section{RESULTS}

The basic reproduction number (Ro) of COVID-19 has been calculated from 60 days of confirmed cases for each SRRAC included country. Since the starting period of COVID-19 is different for each country, we have considered the first 60 days confirmed cases individually where increases are exponential. For calculating the values, Ro of SARS-Co-V-2, Generation Time (GT) must be reached, referred to as the generation interval. We applied the GT of SARS-CoV-2 in our study as GT of SARS-CoV-2 (GT=5.2 days, $S D=1.72$ days). Primarily, all possible combinations of first and last dates were predicted under EG methods (Table 1). In Bangladesh, the optimal time window starting on March 17, 2020, and ending on May 16, 2020, provided the maximum value of Ro was $3.86(95 \% \mathrm{Cl}$ : 3.574.17).Minimum Ro was 1.31 (95\% Cl: 1.26 -1.36) was observed in Bhutan, where the optimal time window was starting on March 6, 2020, and ending on May 5, 2020. In optimal time window on March 8,2020 , to May 7, 2020. The Maldives provides the secondhighest value of Ro was 3.22 (95\% Cl: 2.62 - 4.02). In India, we have found the values of Ro was 2.07 (95\% $\mathrm{Cl}$ : 2.04-2.10), during the optimal time window from March 3, 2020, to May 2, 2020. Afghanistan provides the values of Ro was 1.47 (95\% Cl: $1.45-1.49$ ), within the optimal time window from March 8, 2020, to May 7, 2020. In Pakistan, during the optimal time window from 
February 2, 2020, to May 23, 2020, we got Ro 1.43 (95\% Cl: 1.42-1.44). In Nepal and Sri-Lanka, we observed the values of Ro was $1.75(95 \% \mathrm{Cl}: 1.66-1.84)$ and Ro was 2.39 (95\% Cl: 2.12-2.69).

Sensitivity analysis: The EG method allows the operator to select an exponential time interval over the growth of confirmed cases is exponential. Plate-l: Fig. 1(a)-8(a) shows that confirmed cases are growth exponential for Afghanistan, Bangladesh, Bhutan, India, Maldives, Nepal, Pakistan, and Sri-Lanka, respectively. A better selection can be rendered by taking the Rsquared deviance statistic over several potential periods. The most significant value of R-squared related to periods is that the analysis fits the data best. We choose this time to offer projections. For this period, the task analysis calculates the deviation R-squared statistics with different time intervals selected by the operator. A plot marked as red provides the most massive R-squared valued time intervals of cumulative length obtained from Plate-II: Fig. 1(b)-8(b)for Afghanistan, Bangladesh, Bhutan, India, Maldives, Nepal, Pakistan, and Sri-Lanka respectively. The time window can present the most massive R-square value and corresponding approximations. Plate-III: Fig. 1(c)-8(c) represents the portion of pandemic curves that best fitted exponential growth and estimate the reproductive numbers for SRRAC included countries. The ap- proximation of the reproductive number persisted within the confidence interval $(\mathrm{Cl}) 95 \%$ of the best fit for selecting time intervals, indicating that the calculation was robust for exponential growth season changes.Table-2 also represents the Reproduction rate, Rsquared, and Growth rate of SRRAC countries.

\section{DISCUSSION}

The current season (winter season-2020) of the pandemic in the world is most important, surprisingly, as coronavirus diseases increase. During the winter season, the second coronavirus wave has already reached several countries. In China, estimated values $\mathrm{R}_{0} 2.24(95 \% \mathrm{Cl}: 1.95-2.55)$ of were to $5.71(95 \%$ Cl: 4.24-7.54) (Zhao et al., 2020). In Africa, the estimated value of $\mathrm{R}_{0}$ was $2.37 \quad(95 \% \mathrm{Cl}$ : 2.222.51) (Musa et al., 2020). In our estimation, we have $\mathrm{R}_{0}$ which observed the values of for Bangladesh was $3.86(95 \% \mathrm{Cl}: 3.57-4.17)$, which is the highest in SRRAC countries and near to European regions ( $\mathrm{Xu}$ et al., 2020). This value of $R_{0}$ is predicting us horrible epidemic situation. The second highest have found in the Maldives was 3.22(95\% Cl: 2.62-4.02). In SRRAC included countries, the lowest value of $\mathrm{R}_{0}$ has been found in Bhutan was 1.31 (95\%Cl:1.26-1.36). For India, Pakistan, Afghanistan, Nepal, and Sri-Lanka we got the values of $\mathrm{R}_{0}$ are 2.07$\left.) 95 \% \mathrm{Cl}: 2.04-2.10\right), 1.43$

Table 1. Basic reproduction estimates by Exponential growth (EG) method.

\begin{tabular}{llllll}
\hline \multirow{2}{*}{ Country } & \multicolumn{2}{c}{ Input data duration } & Optimal Time & Default & Optimal \\
\cline { 2 - 3 } \cline { 5 - 6 } & Start & end & Window & $\mathbf{R}_{\mathbf{0}}$ & $\mathbf{R}_{\mathbf{0}}$ \\
\hline Afghanistan & 08.03 .2020 & 07.05 .2020 & $1: 59$ & $1.49[1.47-1.51]$ & $1.47[1.45-1.49]$ \\
Bangladesh & 17.03 .2020 & 16.05 .2020 & $11: 31$ & $3.97[3.70-4.26]$ & $3.86[3.57-4.17]$ \\
Bhutan & 06.03 .2020 & 05.05 .2020 & $4: 35$ & $1.30[1.25-1.35]$ & $1.31[1.26-1.36]$ \\
India & 03.03 .2020 & 02.05 .2020 & $1: 42$ & $2.02[1.10-2.05]$ & $2.07[2.04-2.10]$ \\
Maldives & 08.03 .2020 & 07.05 .2020 & $6: 30$ & $4.69[3.84-5.80]$ & $3.22[2.62-4.02]$ \\
Nepal & 03.24 .2020 & 23.05 .2020 & $1: 59$ & $1.74[1.65-1.83]$ & $1.75[1.66-1.84]$ \\
Pakistan & 27.02 .2020 & 23.05 .2020 & $1: 59$ & $1.43[1.42-1.44]$ & $1.43[1.42-1.44]$ \\
Sri Lanka & 12.03 .2020 & 11.05 .2020 & $28: 47$ & $2.51[2.24-2.81]$ & $2.39[2.12-2.69]$ \\
\hline
\end{tabular}

Table 2. Reproduction rate, R-squared and Growth rate of SRRAC countries.

\begin{tabular}{llll}
\hline Country Name & Reproduction rate (R) & R-Squared & Growth rate \\
\hline Afghanistan & 1.4852 & 0.8847 & 0.0707 \\
Bangladesh & 3.9690 & 0.9447 & 0.2587 \\
Bhutan & 1.2991 & 0.9607 & 0.0465 \\
India & 2.0226 & 0.09264 & 0.1278 \\
Maldives & 4.6891 & 0.7770 & 0.2923 \\
Nepal & 1.7374 & 0.6428 & 0.0995 \\
Pakistan & 1.4333 & 0.7036 & 0.0643 \\
Sri Lanka & 2.5101 & 0.7503 & 0.1689 \\
\hline
\end{tabular}


Uddin M. N. et al. / J. Appl. \& Nat. Sci. 12(4): 628 - 634 (2020)
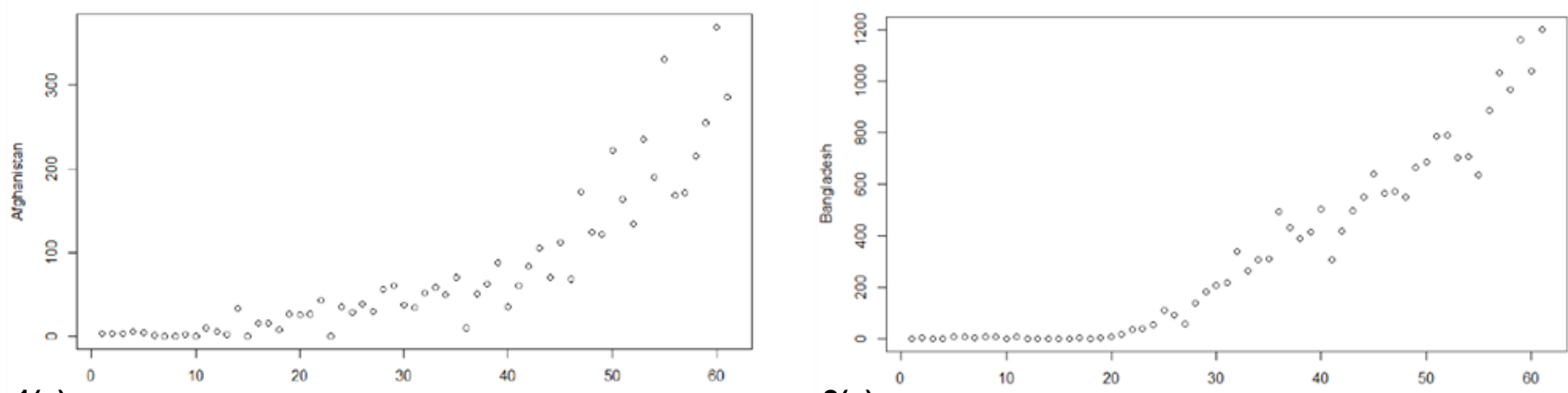

1(a)

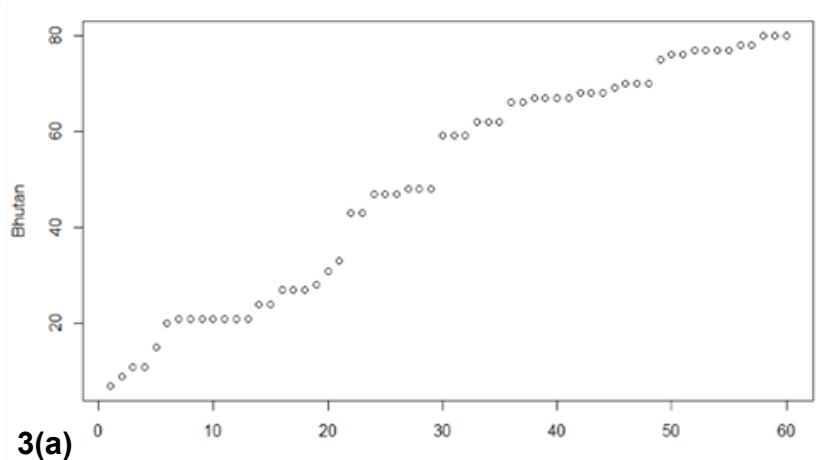

3(a)

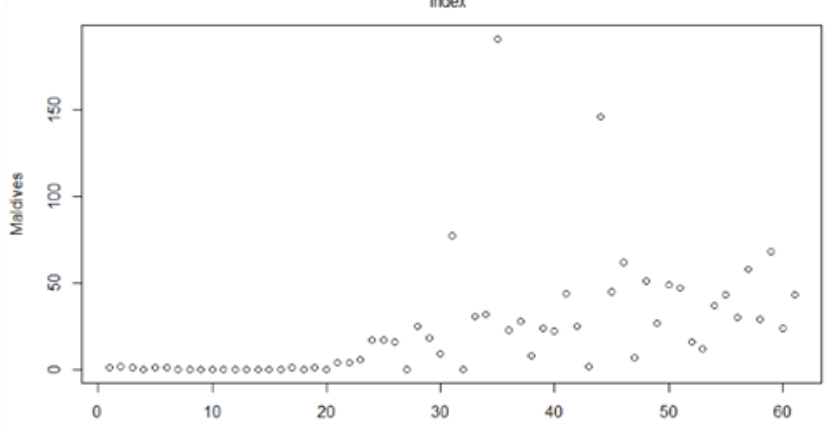

5(a)

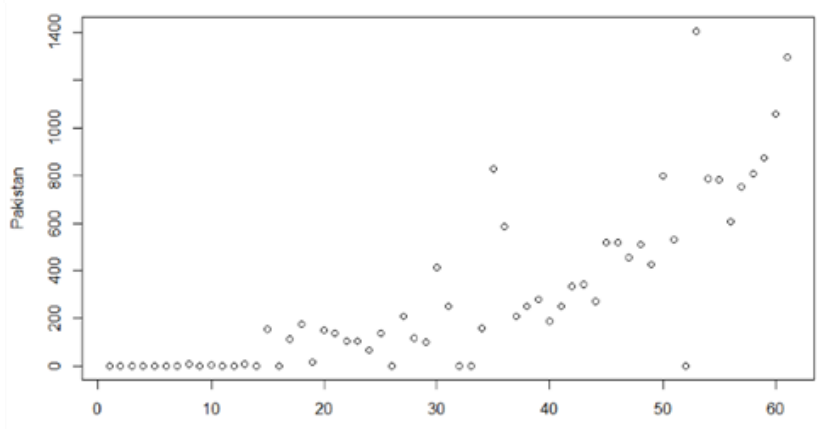

7(a) 2(a)
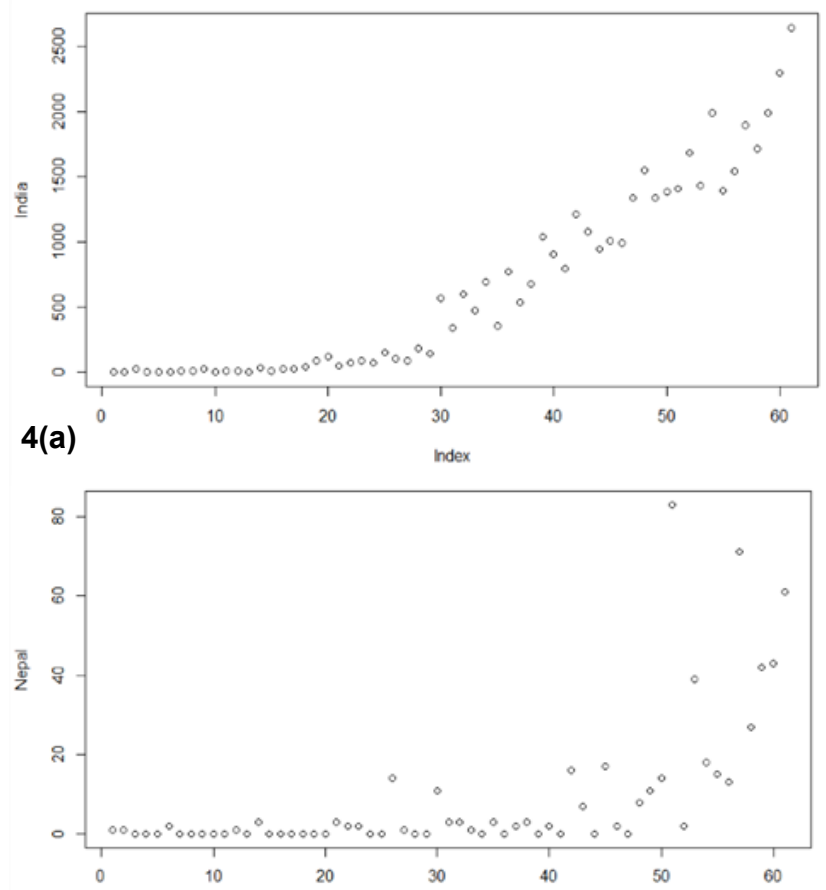

6(a)

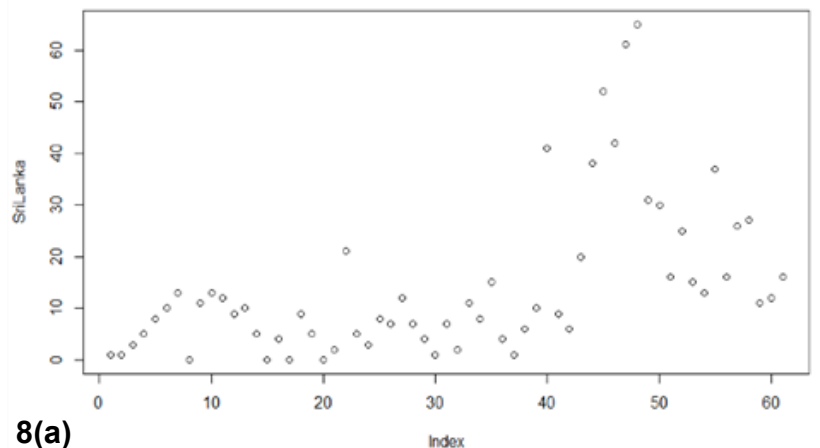

Plate-I: Fig. 1-8. Basic reproduction number estimation for Afghanistan, Bangladesh, Bhutan, India, Maldives, Nepal, Pakistan and Sri-Lanka as shown in Fig. 1(a), 2(a), 3(a), 4(a), 5(a), 6(a), 7(a), and 8(a) respectively by Exponential Growth model where the growth of confirmed cases are exponential.

(95\% $\mathrm{Cl}: 1.42-1.49), 1.75(95 \% \mathrm{Cl}: 1.66-1.84)$ and 2.39 (95\% Cl:2.12-2.69). Here we have found the range of $R_{0} 1.00$ to 4.00 , which are significantly greater than one and indicates the potential of SARS-Co-V-2 to causes an outbreak. As an assumption from our estimation, for high Ro predicate values, the epidemic of COVID-19 would spread rapidly in these countries. This future indicates that South Asians are the most vulnerable for COVID-19. So, we suggest taking serious control measures, public awareness, and other health policies to regulate the spread of COVID-19 in SRRAC included countries. In March, the SAARC platform coordinated the response of countries and formed a SAARC COVID-19 emergency fund to mitigate the virus's adverse consequences, but further coordination and efforts at the regional level are need- 

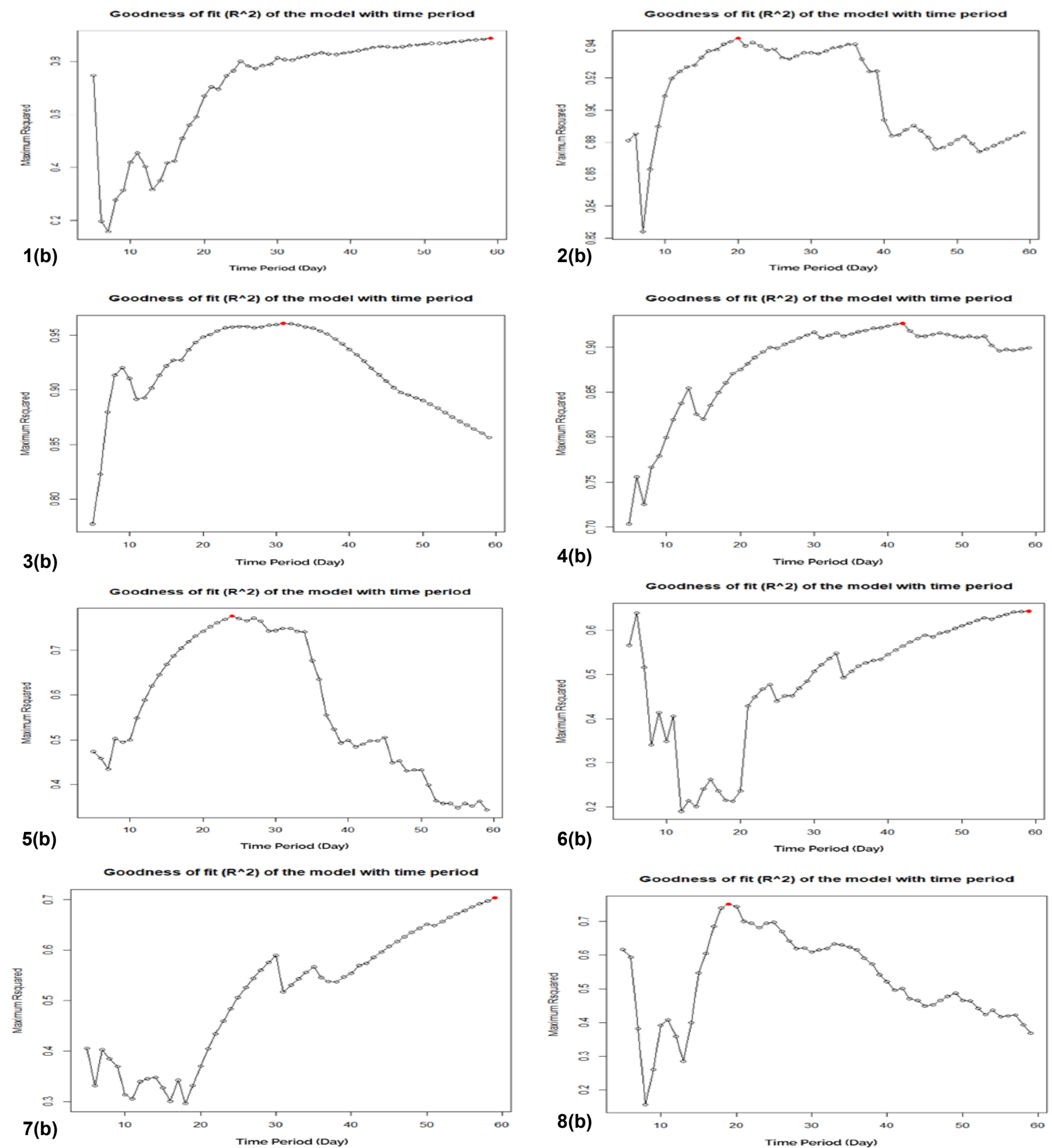

Plate II: Fig. 1-8. Extreme deviance R-squared statistic for time intervals of cumulative length for Afghanistan, Bangladesh, Bhutan, India, Maldives, Nepal, Pakistan and Sri-Lanka as shown in Fig. 1(b), 2(b), 3(b), 4(b), 5(b), 6(b), 7 (b), and 8(b) respectively. The best value is matched by a plot with red color.

ed via active cooperation, mutual support, the sharing of virus containment techniques, joint research programs and regular active surveillance by SAARC countries (Chan et al., 2020).

\section{Conclusion}

In short, the baseline SARS-CoV-2 reproduction number is predicted for each SAARC country using the exponential growth process. We estimated the value within the confidence interval $(\mathrm{Cl})$ of $95 \%$ for each SRRAC included countries. Here, we have observed the range of Ro was 1.00 to 4.00. The determination was significantly higher than 1 , proving that SARS$\mathrm{CoV}-2$ is hugely transmissible. Either vaccination or further preventive control steps should be carried out to decrease the scale of the outbreak. 
Uddin M. N. et al. / J. Appl. \& Nat. Sci. 12(4): 628 - 634 (2020)
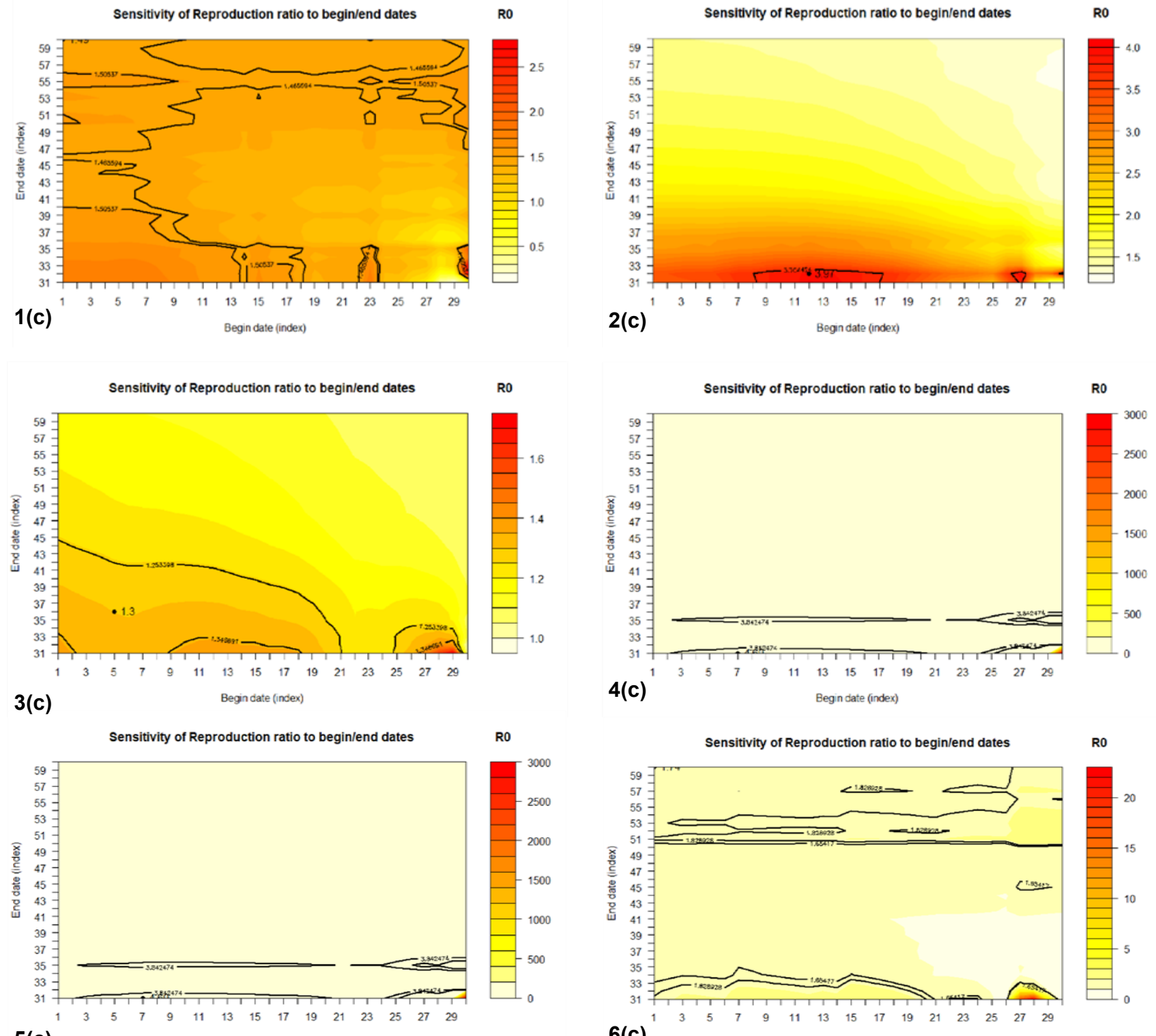

5(c)

$$
\text { Begin date (index) }
$$
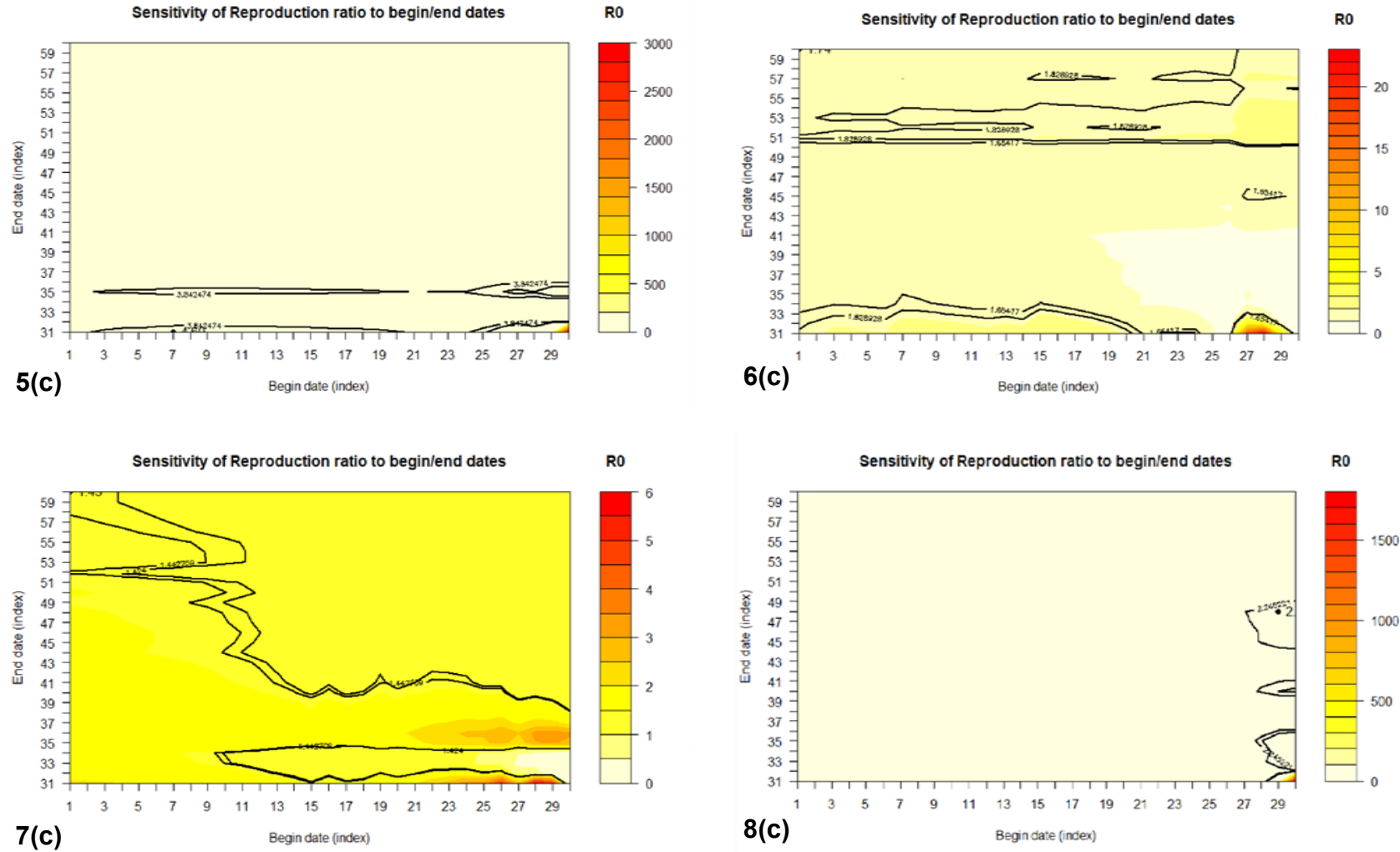

Plate III. Fig. 1-8. Reproductive ratio is approximated by several beginnings and end dates for the period. Fig.1(c), 2(c), 3(c), 4(c), 5(c), 6(c), 7(c), and 8(c) results for Afghanistan, Bangladesh, Bhutan, India, the Maldives, Nepal, Pakistan and Sri-Lanka respectively. A point is marked as the corresponding best fit and the solid black lines reflect the boundary of the equivalent $95 \% \mathrm{Cl}$. An estimation that drops below the $\mathrm{Cl}$ of 95\%, however. A large range of beginning and finishing dates can be obtained from the rate that shows the best match. Such dates are the outcomes once determined between the solid black lines. 


\section{Conflict of interests}

The authors declare that they have no conflict of interests.

\section{REFERENCES}

1. Chan, J. F. W., Yuan, S., Kok, K. H., To, K. K. W., Chu, H., Yang, J., and Tsoi, H. W. (2020). A familial cluster of pneumonia associated with the 2019 novel coronavirus indicating person-to-person transmission: a study of a family cluster. The Lancet, 395 (10223), 514-523.

2. Dey, S. K., Rahman, M. M., Shibly, K. H., Siddiqi, U. R., and Howlader, A. (2020). Epidemic Trend Analysis of SARS-CoV-2 in SAARC Countries Using Modified SIR (M-SIR) Predictive Model. medRxiv.

3. Hasan, M., Hossain, A., Bari, W., and Islam, S. S. (2020). Estimation of the basic reproduction number of novel coronavirus (COVID-19) in Bangladesh: A 65-day outbreak data-driven analysis.

4. Heesterbeek, J. A. P. (2002). A brief history of R 0 and a recipe for its calculation. Acta biotheoretica, 50 (3), 189-204.

5. Liu, T., Hu, J., Kang, M., Lin, L., Zhong, H., Xiao, J., and Deng, A. (2020). Transmission dynamics of 2019 novel coronavirus (2019-nCoV) doi: https:// doi.org/10.1101/2020.01.25.919787

6. Musa, S. S., Zhao, S., Wang, M. H., Habib, A. G., Mustapha, U. T., and He, D. (2020). Estimation of exponential growth rate and basic reproduction number of the coronavirus disease 2019 (COVID19) in Africa. Infect Dis Poverty, 9 (1):96

7. Mustafa, F., Sherwani, R. A. K., Saqlain, S. S., Meraj, M. A., and Ayyaz, R. (2020). COVID-19 in South Asia: Real-time monitoring of reproduction and case fatality rate. arXiv preprint arXiv:2008.04347

8. Obadia, T., Haneef, R., and Boëlle, P. Y. (2012). The R0 package: a toolbox to estimate reproduction numbers for epidemic outbreaks. BMC Medical Informatics and Decision Making, 12 (1): 1-9

9. Wallinga, J., and Lipsitch, M. (2007). How generation intervals shape the relationship between growth rates and reproductive numbers. Proceedings of the Royal Society B: Biological Sciences, 274 (1609): 599-604

10.Xu, C., Dong, Y., Yu, X., Wang, H., Tsamlag, L., Hang, S., Chang, R., Wang,Z., Yu, Y, Long, R.,Wang, Y., Gang Xu, G., Shen, T., Wang, S., Zhang, X., Wang, H and Cai, Y. (2020). Estimation of reproduction numbers of COVID-19 in typical countries and epidemic trends under different prevention and control scenarios. Frontiers of Medicine, $14: 613-622$

11.Zhao, S., Lin, Q., Ran, J., Musa, S. S., Yang, G., Wang, W., and Wang, M. H. (2020). Preliminary estimation of the basic reproduction number of novel coronavirus (2019-nCoV) in China, from 2019 to 2020: A data-driven analysis in the early phase of the outbreak. International Journal of Infectious Diseases, 92: 214-217 